\title{
Research on RHT constitutive model parameters of fiber reinforced concrete based on experiment and numerical simulation
}

\author{
Shuaishuai Wang ${ }^{1, a}$, Luhui Yan ${ }^{1, b}$, Qiaoheng Liang ${ }^{2, \mathrm{c}}$ and Mohan $\mathrm{Xu}{ }^{1, \mathrm{~d}}$ \\ ${ }^{1}$ The College of Basic Education, NUDT, Changsha, China \\ ${ }^{2}$ Special police academy, Beijing, China \\ atzwwss@163.com, ${ }^{b}$ yan I h@aliyun.com, ${ }^{9}$ lahafx@163.com, ${ }^{d}$ xmh19419@163.com
}

\begin{abstract}
Keywords: The RHT constitutive model; UHMWPE fiber reinforced concrete; Experiment; Nu -merical simulation

Abstract. In this paper, a method for determining the parameters of the RHT material model, which can hardly be determined by total experiments, based on the combination of experiment and numerical simulation is proposed. The determination process and verification are analyzed taking UHMWPE fiber reinforced concrete as an example. The result shows that the obtained parameters can relatively well reflect the mechanical properties of the fiber reinforced concrete, which proves the effectiveness of the method.
\end{abstract}

\section{Introduction}

Concrete is one of the most common engineering materials. In order to improve the abilities of military protective engineering resisting to impact and penetration, recently people focuse on the development of the high-performance concrete reinforced by fibers. Many penetration researches on these materials were carried out $[1,2]$.

The dynamic models of materials under complicated stress state are needed for simulation study for the impact dynamic problems of fiber reinforced concrete. The HJC model [3], the RHT model [4], the LLNT model [5] and the Malvar model [6] are among the most popular dynamic models. The RHT model [7], modelled by three strength limit surfaces, developed on the basis of the HJC model. Taking the difference of tensile, compressive strength and strain rate sensitivity into account, the model can reflect the dynamic mechanical behavior of concrete material more completely. Because of that, the RHT model has become the most worthy of consideration for cement-based material. However, this brings difficulty to the determination of parameters as well [8]. Z.Tu et al. [9] calculated the damage parameters of the RHT model, and found that the numerical simulation with the parameter values given by AutoDyn would cause unexpected strain softening. A further modification of the material parameters $A, N, Q_{0}$ and $B_{\mathrm{Q}}$ were made by R.Q. Zhang, according to the expression of the failure strength surface and experiment data. It is pointed out that the modification process can be applied to other specific concrete materials.

Generally speaking, the RHT model has many parameters and the determination of those parameters needs different kinds of material experiments. To simplify the experimental system for parameters, a determination method based on the combination of experiment and simulation is proposed, taking UHNWPE fiber reinforced concrete as an example. The main parameters can be determined according to the dynamic and static mechanical experiment. The rest parameters then will be checked and adjusted through the numerical simulation of relevant experiments. Finally, reasonable parameters for a specific concrete can be obtained.

\section{Introduction to the RHT Model}

The RHT model considers three ultimate surfaces, namely, failure surface, elastic limit surface and residual strength surface (Fig. 1), describing the failure strength, initial yield strength and residual strength of the concrete respectively [7].

The equation of the failure plane is expressed as a function of hydrostatic pressure $p$, Lode angle $\theta$ and strain rate $8 \delta$ : 

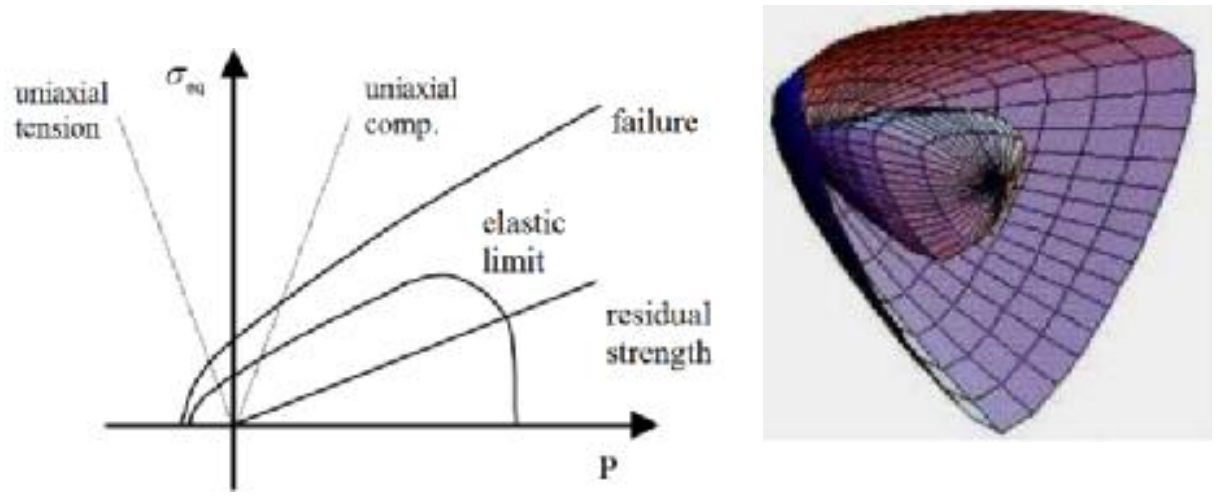

Fig. 1 Meridian and three-dimensional images of the RHT model

$$
\sigma_{\mathrm{eq}}^{*}(p, \theta, 8 \&)=Y_{\mathrm{TXC}}^{*}(p) \cdot R_{3}(\theta) \cdot F_{\mathrm{RATE}}(8),
$$

where $\sigma_{\text {eq }}^{*}$ is normalized equivalent stress;

$F_{\text {RATE }}(\&)$ is strain hardening factor, expressed as

$$
F_{\text {RATE }}(\&)=\left\{\begin{array}{lllll}
\left(\frac{8 \&}{8 \&}\right)^{\alpha} & \text { for } & p>\frac{f_{\mathrm{c}}}{3} & \text { with } & \& \&=30 \times 10^{-6} \mathrm{~s}^{-1} \\
\left(\frac{\& \&}{8 \&}\right)^{\delta} & \text { for } & p<\frac{f_{\mathrm{t}}}{3} & \text { with } & \& \&=3 \times 10^{-6} \mathrm{~s}^{-1}
\end{array} .\right.
$$

$Y_{\mathrm{TXC}}^{*}(p)$ represents the compressive meridian, given by

$$
Y_{\mathrm{TXC}}^{*}(p)=A\left[p^{*}-p_{\text {spall }}^{*} F_{\mathrm{RATE}}(8)\right]^{N},
$$

where $p^{*}=p / f_{\mathrm{c}}$ is normalized hydrostatic pressure ( $f_{\mathrm{c}}$ is the axial compressive strength), and $p_{\text {spall }}^{*}=p_{\text {spall }} / f_{\mathrm{c}}$ is normalized spalling strength. $A, N$ is the strength parameters of the compressive meridian, which can be obtained by using the experiment data of conventional triaxial compression.

The function of Lode angle $R_{3}(\theta)$ representing the relationship among the failure strength of concrete, the second and third invariants of deviatoric stress tensor and hydrostatic pressure, is expressed as

$$
R_{3}(\theta)=\frac{2\left(1-Q_{2}^{2}\right) \cos \theta+\left(2 Q_{2}-1\right)\left[4\left(1-Q_{2}^{2}\right) \cos ^{2} \theta+5 Q_{2}^{2}-4 Q_{2}\right]^{\frac{1}{2}}}{4\left(1-Q_{2}^{2}\right) \cos ^{2} \theta+\left(1-2 Q_{2}\right)^{2}}
$$

where $Q_{2}$ is the portion of the equivalent strength $r_{\mathrm{t}}$ on the tensile meridian to the strength of triaxial compression $r_{\mathrm{c}}$, and

$$
\begin{array}{ll}
\theta=\frac{1}{3} \cos ^{-1}\left[\frac{3 \sqrt{3} J_{3}}{2 J_{2}^{1 / 2}}\right] & 0 \leq \theta \leq \frac{\pi}{3}, \\
Q_{2}=\frac{r_{\mathrm{t}}}{r_{\mathrm{c}}}=Q_{0}+B_{\mathrm{Q}} p^{*} & 0.51 \leq Q_{2} \leq 1.0 .
\end{array}
$$


It is clear that $R_{3}(\theta)$ is determined by $Q_{0}$ and $B_{\mathrm{q}}$, where $Q_{0}$ is the ratio of the tensile and compressive strength in the $\pi$ plane, and $B_{\mathrm{q}}$ reflects a brittle-to-ductile transition.

The elastic limit plane is defined as follows, where the cap function is related to the material elastic limit and the normalized hydrostatic pressure.

$$
Y_{\text {ela }}^{*}=Y_{\text {fail }}^{*} F_{\mathrm{CAP}}(P) .
$$

$B$ and $M$ are the parameters of the residual strength surface, defined as follows:

$$
Y_{\text {res }}^{*}=B \cdot\left(p^{*}\right)^{M}
$$

$B$ and $M$ can be obtained according to the normalized residual strength from relevant experiments and Eq. 8.

\section{Determination of the RHT model parameters based on experiment}

Determination of spalling strength. In the current study of the RHT model parameters, the value of $p_{\text {spall }}^{*}$ was usually given the default value $0.1[10,11]$. Since the static spalling strength is the strength under confined uniaxial tension, it is difficult to design an experiment. However the static spalling strength has a relationship with the uniaxial tensile strength.

The strain components except $\varepsilon_{x}$ are fixed to zero under confined uniaxial deformation state. According to the elastic theory, there is

$$
\sigma_{x}=(\lambda+2 \mu) \varepsilon_{x}=E_{1} \varepsilon_{x},
$$

where $\lambda$ and $\mu$ are Lame constants, $\lambda=\frac{E v}{(1+v)(1-2 v)}$ and $\mu=\frac{E}{2(1+v)}$ (E and $v$ are the elastic modulus and Poisson's ratio respectively).

Under uniaxial tension, the relationship between the tensile limit $f_{\mathrm{t}}$ and the ultimate tensile strain is $\varepsilon_{\mathrm{t}}=\frac{f_{\mathrm{t}}}{E}$. When the crack happens, the strain is $\varepsilon_{\mathrm{t}}$, and $p_{\text {spall }}=(\lambda+2 \mu) \varepsilon_{\mathrm{t}}$. Then the $p_{\text {spall }}^{*}$ can be solved.

The spalling strength of the UHMWPE fiber reinforced concrete was calculated as shown in Table 1 based on the results of static split tensile experiment and axial compression experiment [12], where $V_{\mathrm{f}}$ in the table represents the fiber volume fraction.

Table 1 Experiment results and calculated spalling strength of UHMWPE fiber reinforced concrete

(the provision of the compression is positive)

\begin{tabular}{cccccccc}
\hline$V_{\mathrm{f}}$ & $f_{\mathrm{t}}(\mathrm{MPa})$ & $f_{\mathrm{c}}(\mathrm{MPa})$ & $E(\mathrm{GPa})$ & $v$ & $E_{1}(\mathrm{GPa})$ & $p_{\text {spall }}(\mathrm{MPa})$ & $p_{\text {spall }}^{*}$ \\
\hline 0 & -3.55 & 53.2 & 34.78 & 0.21 & 39.1510 & 3.9061 & -0.0751 \\
0.3 & -5.23 & 52.9 & 32.63 & 0.23 & 37.8276 & 6.0631 & -0.1146 \\
0.5 & -5.44 & 47.3 & 29.60 & 0.26 & 36.2169 & 6.6561 & -0.1407 \\
0.7 & -5.58 & 49.2 & 28.66 & 0.27 & 35.8127 & 6.9726 & -0.1417 \\
\hline
\end{tabular}

Determination of failure surface parameters $A$ and $N$. The conventional triaxial compression experiment of the UHMWPE fiber reinforced concrete conducted by researchers [13] represents reliable data for the calculation of compression meridian, where

$$
\sigma_{\mathrm{eq}}^{*}(p)=A\left[p^{*}-p_{\mathrm{spall}}^{*}\right]^{N}
$$


The experiment data [13] were substituded into Eq. 10 for curve fitting using Matlab as shown in Fig. 2. Table 2 shows the values of parameters $A$ and $N$ resulted from the fitting, where $\mathrm{R}^{2}$ represents the correlation coefficient.

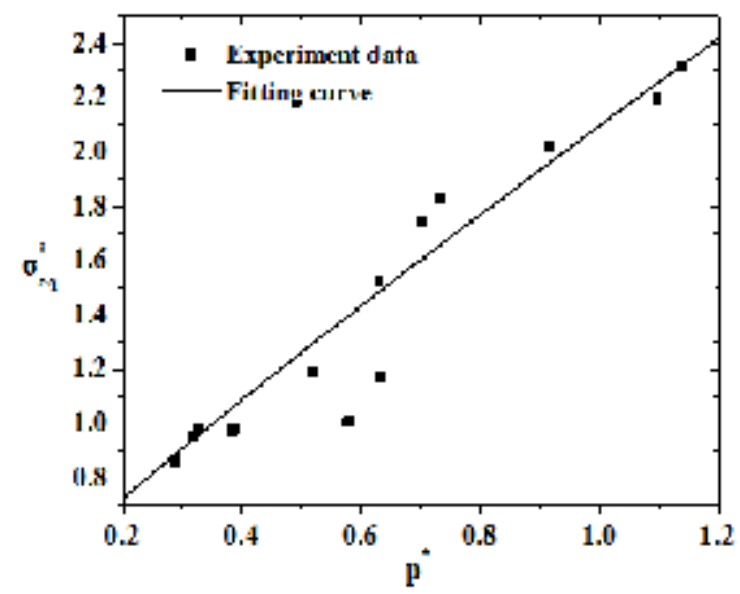

Fig. $2 \sigma_{\mathrm{eq}}^{*}-p^{*}$ 错误!未找到引用源。 fitting curves of fiber reinforced concrete

Table 2 Fitting results of parameters $A$ and $N$

\begin{tabular}{cccc}
\hline$V_{\mathrm{f}}$ & $A$ & $N$ & $R^{2}$ \\
\hline 0 & 2.103 & 0.8776 & 0.9714 \\
0.3 & 1.557 & 0.6934 & 0.8801 \\
0.5 & 1.625 & 0.7670 & 0.9495 \\
0.7 & 1.864 & 0.8811 & 0.9732
\end{tabular}

Determination of parameters $\boldsymbol{Q}_{\mathbf{0}}$ and $\boldsymbol{B}_{\mathbf{Q}}$. According to Eq. $6, Q_{2}=Q_{0}$ when $\mathrm{p}^{*}=0$, then $Q_{0}=$ $r_{\mathrm{t} 0} / r_{\mathrm{c} 0}$. As seen in Eq. 11, the partial stress $r_{\mathrm{c} 0}$ on the compression meridian in $\pi$ plane can be solved by the normalized spalling strength $p_{\text {spall }}^{*}$ and $A, N$ obtained above.

$$
r_{\mathrm{c} 0}=\frac{\sqrt{2} f_{\mathrm{c}}}{3} A \cdot p_{\mathrm{spall}}^{*}
$$

Table 3 gives the calculated $r_{\mathrm{c} 0}$ for each fiber volume fraction:

Table $3 r_{c 0}$ at each fiber volume fraction

\begin{tabular}{ccccc}
\hline$V_{\mathrm{f}} / \%$ & 0 & 0.3 & 0.5 & 0.7 \\
\hline$r_{\mathrm{c} 0}$ & 4.9741 & 6.4356 & 7.0329 & 6.5772 \\
\hline
\end{tabular}

For the lack of experiment related to tensile meridian, we assumed that the meridian is linear before the $\pi$ plane. Then a linear fitting of the split tensile strength [12] with the spalling strength was used to solve the partial stress $r_{\mathrm{t} 0}$ on the tensile meridian in the $\pi$ plane. Since we got $r_{\mathrm{c} 0}$ and $r_{\mathrm{t} 0}$, $Q_{0}$ was solved.

On the tensile meridian, $R_{3}(\theta)$ can be simply substituded by $Q_{2}$, and the equation of the failure plane is changed to

$$
\sigma_{\text {eq }}^{*}(p, \theta)=A\left[p^{*}-p_{\text {spall }}^{*}\right]^{N}\left(Q_{0}+B_{\mathrm{q}} p^{*}\right)
$$

Noticed that the spalling strength also locates on the meridian meridian, then $B_{\mathrm{Q}}$ can be obtained by substituding calculated spalling strength combined with parameters $A, N$, and $Q_{0}$ into the equation of the tensile meridian. Table 4 shows the final calculation results of $Q_{0}$ and $B_{\mathrm{Q}}$. 
Table 4 Calculation results of parameters $Q_{0}$ and $B_{\mathrm{Q}}$

\begin{tabular}{ccc}
\hline$V_{\mathrm{f}}$ & $Q_{0}$ & $B_{\mathrm{Q}}$ \\
\hline 0 & 0.3011 & 0.9181 \\
0.3 & 0.3316 & 1.4200 \\
0.5 & 0.4170 & 1.0454 \\
0.7 & 0.6483 & 0.2652 \\
\hline
\end{tabular}

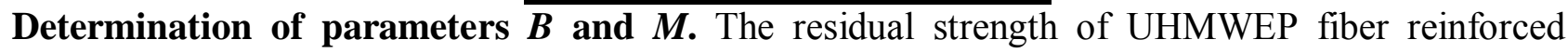
concrete under conventional triaxial compression were substituded into Eq. 8 and fitted by Matlab as shown in Fig. 3. Table 5 shows the results of $B$ and $M$, where $\mathrm{R}^{2}$ is the correlation coefficient.

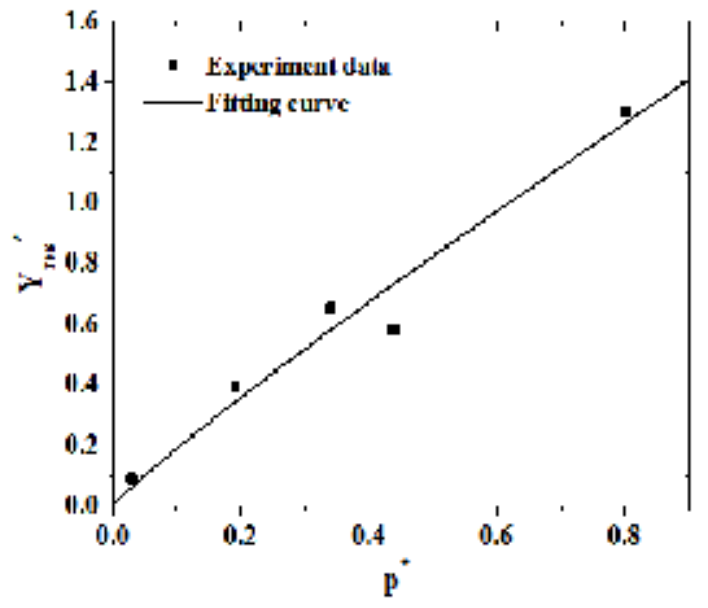

Fig. $3 Y_{r e s}^{*}$ 错误!未找到引用源。fitting curves of fiber reinforced concrete

\begin{tabular}{cccc}
\multicolumn{4}{c}{ Table 5 Fitting results of parameters $B$ and $M$} \\
\hline$V_{\mathrm{f}}$ & $B$ & $M$ & $R^{2}$ \\
\hline 0 & 1.884 & 1.367 & 0.8937 \\
0.3 & 1.081 & 0.714 & 0.7494 \\
0.5 & 1.025 & 0.446 & 0.7618 \\
0.7 & 1.548 & 0.909 & 0.9604
\end{tabular}

Determination of dynamic strain rate index $\boldsymbol{\alpha}$ and $\delta$. The dynamic strain rate index includes compressive strain rate index $\alpha$ and tensile strain rate index $\delta$, which can be solved according to the dynamic compression and split experiment [12].

The equation of the failure surface under static condition is

$$
\sigma_{\text {eqs }}^{*}(p, \theta)=A\left[p^{*}-p_{\text {spall }}^{*}\right]^{N} \cdot R_{3}(\theta) \text {. }
$$

Under the dynamic condition, the failure surface equation is ( $F_{\text {RATE }}(\&)$, see Eq. 2$)$

$$
\sigma_{\text {eqt }}^{*}(p, \theta)=A\left[p^{*}-p_{\text {spall }}^{*} \cdot F_{\mathrm{RATE}}(\& \&]^{N} \cdot R_{3}(\theta) \cdot F_{\mathrm{RATE}}(\& \&),\right.
$$

Then the dynamic increase factor DIF can be expressed as

$$
D I F=\left[p^{*}-p_{\text {spall }}^{*} \cdot F_{\mathrm{RATE}}(8)\right]^{N} \cdot F_{\mathrm{RATE}}(\&)
$$

where $p^{*}=\frac{p^{*}}{p^{*}-p_{\text {spall }}^{*}}$ and $p_{\text {spall }}^{*}=\frac{p_{\text {spall }}^{*}}{p^{*}-p_{\text {spall }}^{*}}$. 
The DIF extracted from the relevant experiments [12] were substituted into Eq. 14 to solve the two parameters. The obtained results are shown in Table 6.

Table 6 Calculation results of the strain rate index $\alpha$ and $\delta$

\begin{tabular}{ccc}
\hline$V_{\mathrm{f}} / \%$ & $\alpha$ & $\delta$ \\
\hline 0 & 0.0208 & 0.0883 \\
0.3 & 0.0168 & 0.0829 \\
0.5 & 0.0222 & 0.0771 \\
0.7 & 0.0179 & 0.0752 \\
\hline
\end{tabular}

\section{Correction of the RHT model parameters based on simulation}

Finite element model. The numerical simulation of UHMWPE fiber reinforced concrete triaxial compression experiment [13] was carried out by using finite element software Autodyn. The Lagrangian algorithm was used to establish a 1: 1 cylindrical model (Fig. 4a). The z-direction displacement of the upper surface of the model was fixed to 0 (Fig. $4 \mathrm{~b}$ ) and an axial displacement was applied to the bottom surface of the model, with a loading speed of $0.017 \mathrm{~m} / \mathrm{s}$ (Fig. 4c), which was consistent with the experimental. A constant confining pressure was applied to the side surface of the model (Fig. 4d) according to the experiment.
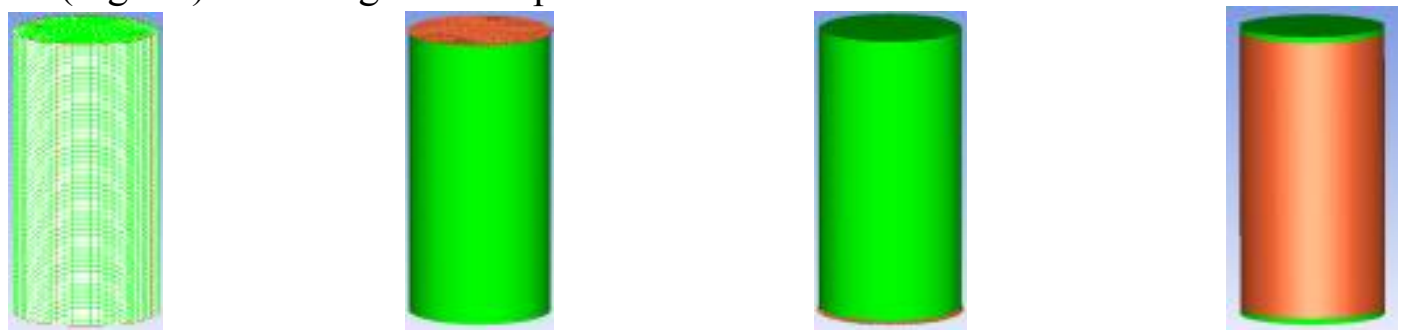

(a) Element model (b) Displacement boundary (c) Loading boundary (d) Confining boundary

Fig. 4 Simulation model of triaxial compression experiment

Simulation results and parameters correction. Figure 5 shows the comparison of failure mode between simulation and experiment based on the numerical cloud chart of damage. As there were 4 kinds of fiber volume fractions $(0 \%, 0.3 \%, 0.5 \%, 0.7 \%)$, and 5 kinds of confining pressure (0MPA, 3MPA, 6MPA, 12MPA, 18MPA), the total number of specimens was 20 , indicating the difficulty to cover all of them in this paper.

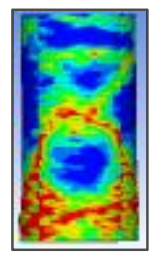

(a) simulation $(0 \mathrm{MPa})$

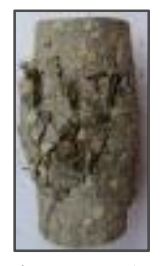

(b) experiment $(0 \mathrm{MPa})(\mathrm{c})$ simulation $(3 \mathrm{MPa})$
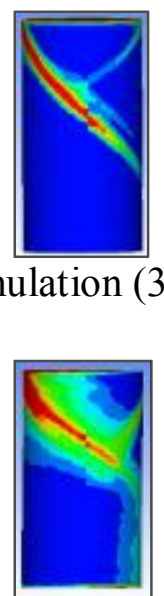

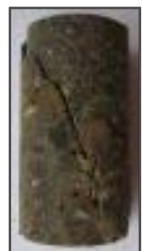

(d) experiment $(3 \mathrm{MPa})$
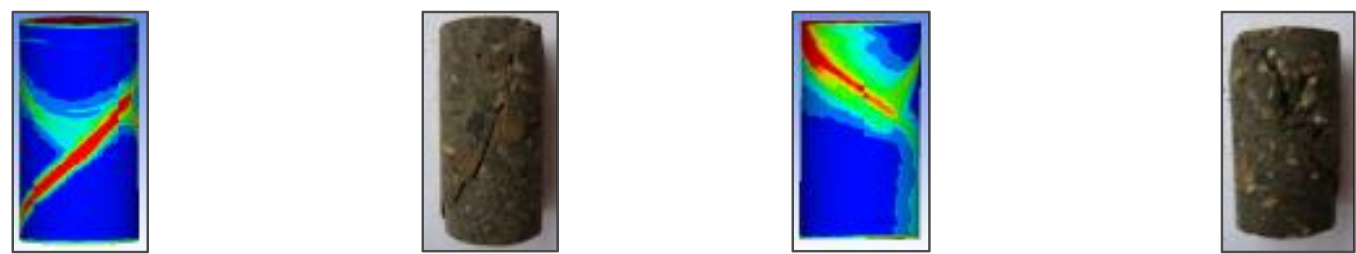

(e) simulation (6 MPa) (f) experiment $(6 \mathrm{MPa})(\mathrm{g})$ simulation $(12 \mathrm{MPa})(\mathrm{h})$ experiment $(12 \mathrm{MPa})$

Fig. 5 Comparison of failure mode between simulation and experiment

Here we only choose the concrete with fiber volume fraction of $0.7 \%$ under the confining pressure from 0 to $12 \mathrm{MPa}$. The simulation results of the concrete with other fiber volume fractions are similar. 
Fig. 6 shows the simulation results of stress-strain responses of the concrete with different fiber volume fractions under confining pressure of $18 \mathrm{MPa}$, and the simulation results under other confining pressures are similar. The results represent that the curves are in relatively good agreement with the experimental results in the aspect of peak stress and the corresponding strain, indicating a relatively good response of the calculated material model parameters to the material properties. However, the result curves tend to have a longer period of high stress compared to the experiment. Further analysis of the parameter characteristics of the model indicates that the main reason for this problem may be the deviation in the determination of parameters $Q_{0}, B$ and $M$, which needs further corrections.

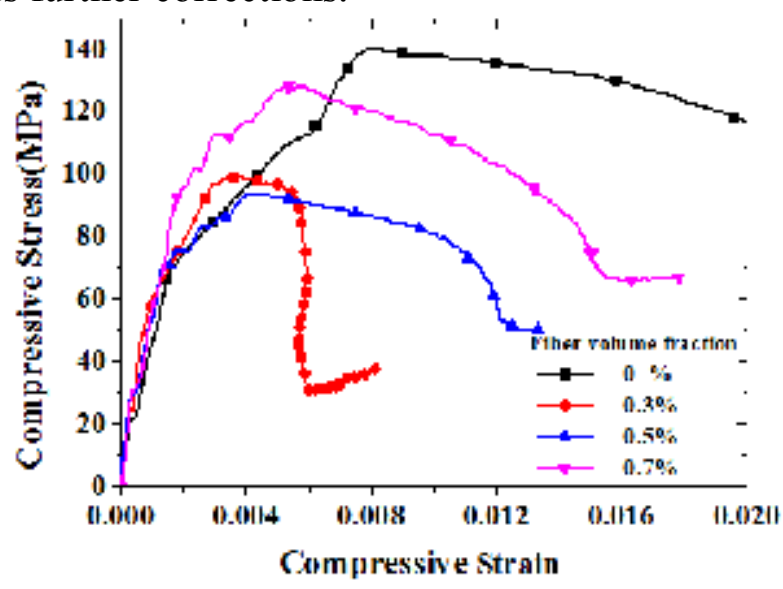

(a) Simulation results

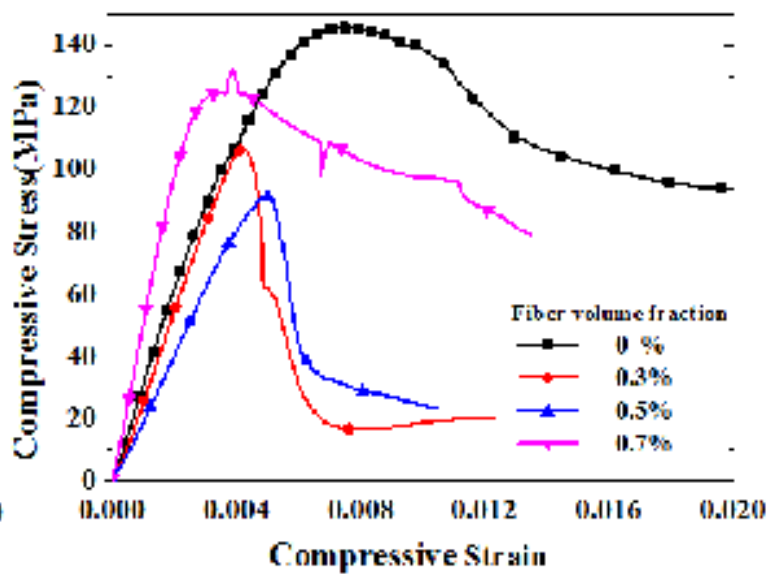

(b) Experiment results

Fig. 6 Comparison of stress-strain responses between simulation and experiment at various fiber volume fractions under confining pressure of $18 \mathrm{MPa}$

For the above deviation, the three parameters were debugged and modified by repeated simulation. The corrected parameters are listed in Table 7.

Table 7 Corrected values of parameters $Q_{0}, B$ and $M$

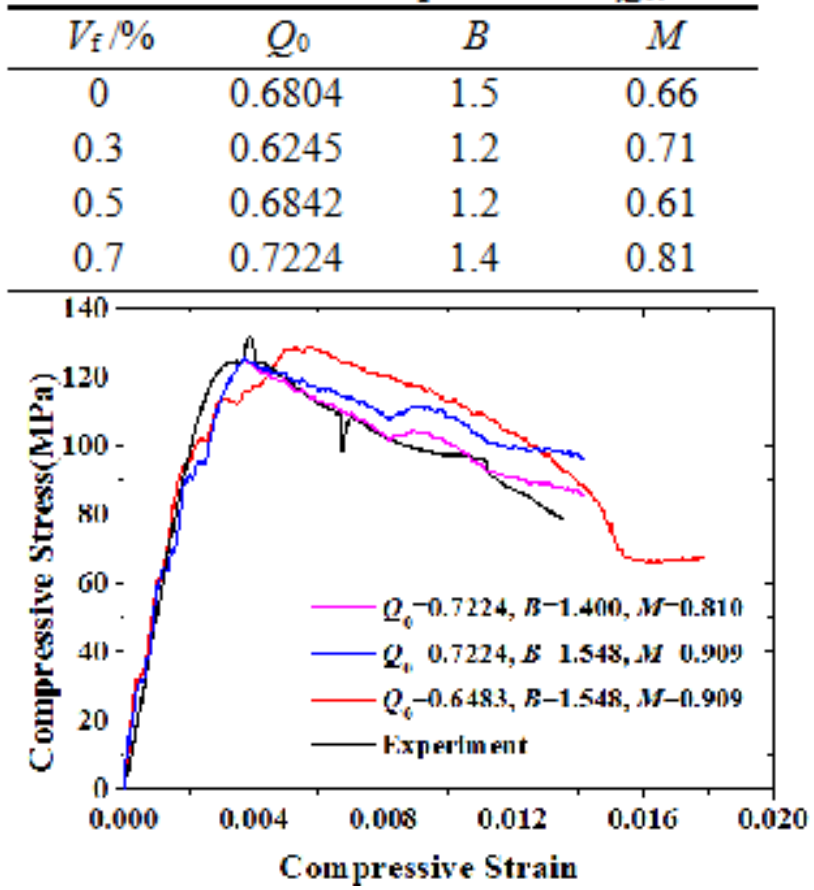

Fig. 7 Comparison of the stress-strain responses between simulation and experiment before and after correction

Fig. 7 shows the comparison of the stress-strain responses between simulation and experiment before and after correction (using fiber volume fraction of $0.7 \%$ and confining pressure of $18 \mathrm{MPa}$ as an example). It is clear that when $Q_{0}=0.7224, B=1.400$ and $M=0.810$, we got the best simulation results. 


\section{Conclusions}

A method for determination of the RHT parameters based on the static and dynamic mechanical experiments and the numerical simulation is proposed in this paper. The result shows that the obtained parameters can relatively well reflect the mechanical properties of fiber reinforced concrete, which proves the effectiveness of the method.

\section{References}

[1] X.P. Li, K. Liu, J.H. Huang et al., Study on anti-penetration performance of steel fiber reinforced high strength concrete without coarse aggregate, Journal of Functional Materials, vol. 48 (2017), p. 22-27.

[2] B. Sun, and Z.Y. Lin, Calculation method for the penetration depth of steel-fiber concrete, Journal of Vibration and Shock, vol. 36 (2017), p. 203-206.

[3] Q.R. Sun, R.Y. Li, Y.Y. Zhao et al., Investigation on parameters of HJC model applied to simulate perforation experiments of reinforced concrete, Engineering Mechanics (2016), p. 248-256.

[4] X.Q. Zhou, and J.Z. ZHu, Numerical simulation of dynamic responses of RC walls under blast loading, Journal of disaster prevention and mitigation engineering, vol. 36 (2016), p. 153-158.

[5] L.S.T. Corporation, LS-DYNA keyword user' s manual, volume II, material models, version 971, California: LSTC, vol. 1 (2007), p. 1-544.

[6] T. Fang, H.L. Wang, Y.J. Luo et al., Bonded slip test and constitutive model of BFRP ribs, Low Temperature Architecture Technology, vol. 07 (2015), p. 6-8.

[7] R. W, T. K, H. S et al., Penetration of reinforced concrete by BETA-B-500, numerical analysis using a new macroscopic concrete model for hydrocodes, Proc. (CD-ROM) 9. Internationales Symposium, Interaction of the Effects of Munitions with Structures, Berlin Strausberg,, vol. 3(7) (1999), p. 315-322.

[8] C. Ji, Y. Long, and W.Q. Wan, Numerical simulation of projectile penetrating into steel fiber reinforced Concrete, Journal of PLA University of Science and Technology, vol. 6(5) (2005), p. 460-463.

[9] Z.G. Tu, and Y. Lu, Evaluation of typical concrete material models used inhydrocodes for high dynamic response simulations, International Journal of Impact Engineering, vol. 36(1) (2009), p. 132-146.

[10] R.Q. Zhang, Y.Q. Ding, and W.H. Tang, Failure strength parameters of HJC and RHT concrete constitutive models Chinese Journal of High Pressure Physics, vol. 25(1) (2011), p. $15-22$.

[11] Z.P. Kuang, and X.K. Yuan, Analysis and simulation of strength parameters of RHT concrete constitutive model, Chinese Quarterly of Mechanics, vol. 33 (1) (2012), p. 158-163.

[12] Y.W. Zhang, Study on static and dynamic mechanical properties of UHMWPE fiber reinforced concrete, National University of Defense Technology, Changsha, 2014.

[13] L.F. Li, L.H. Yan, and Y.W. Zhang, Experiment research of UHMWPE fiber reinforced concrete under triaxial compression. p. 1847-1852. 